\title{
Identifying Ontario geographic regions to assess adults who present to hospital with laboratory-defined conditions: a descriptive study
}

\author{
Carina Iskander BMSc, Eric McArthur MSc, Danielle M. Nash MSc PhD, Sonja Gandhi-Banga PhD, \\ Matthew A. Weir MD MSc, Flory Tsobo Muanda MD PhD, Amit X. Garg MD PhD
}

Abstract

Background: In 2007, an electronic repository called the Ontario Laboratories Information System (OLIS) was introduced to allow health care providers timely access to laboratory test results. Since not all laboratories began submitting their data to OLIS simultaneously, we sought to create a date-dependent table of geographic regions (forward sortation areas [FSAs]) from which people would likely present to a hospital linked to OLIS.

Methods: In this descriptive study, we used administrative data to capture adults in Ontario who presented to the emergency department for any reason from 2007 to 2017 . To assess changes over time, we classified all emergency department visits into fiscal quarters. The primary outcome measure was the proportion of people in a given FSA presenting to an emergency department at an OLIS-linked hospital (v. a hospital not linked to OLIS). To be included in the catchment area, at least $90 \%$ of all emergency department visits in a given quarter from a given FSA must have occurred at an OLIS-linked hospital.

Results: By Dec. 31, 2017, 323 (61.4\%) of 526 Ontario FSAs were in the catchment area (a population of about 8.5 million). There were no differences in selected demographic characteristics or comorbidities between people residing within the catchment area of OLIS-linked hospitals and those residing in the catchment area of unlinked hospitals on Dec. 31, 2017. We used the FSA information to construct a date-dependent table of geographic areas likely to have hospital laboratory data available in OLIS for future studies.

Interpretation: We identified relevant Ontario geographic regions from which people would likely present to a hospital linked to OLIS. These geographic regions constitute a catchment area that may be used in future studies to capture adults who present to an OLIS-linked hospital with laboratory-defined conditions such as acute kidney injury, hyperkalemia and hyponatremia.

\lceil] ealth administrative databases are increasingly being used for population-based studies. ${ }^{1}$ Typically, outcomes for these studies are assessed with the use of diagnostic codes, which have limited accuracy for the identification of some laboratory-diagnosed conditions. ${ }^{2-4}$ This may lead to nondifferential outcome misclassification bias, which underestimates the outcome in these studies. $^{2}$ In Ontario, an important improvement in the diagnostic accuracy of laboratory-diagnosed conditions occurred in 2007 with the introduction of the Ontario Laboratories Information System (OLIS), an electronic repository of the province's laboratory test results. ${ }^{5}$ This system was implemented to allow health care providers timely access to laboratory test results from both community- and hospital-based laboratories. The OLIS data have recently been linked to Ontario's other health care administrative databases at ICES (a not-for-profit research institute; ices.on.ca), which pro- vides opportunities for more accurate assessment of laboratory-diagnosed outcomes such as acute kidney injury, hyperkalemia and hyponatremia.

However, after the implementation of OLIS, not all laboratories began submitting their data simultaneously: community laboratories began their contributions to OLIS from the outset, but hospital-based laboratories across the province began their contributions at various times since 2007, and, as of September 2018, not all had contributed. ${ }^{6}$

\section{Competing interests: None declared.}

This article has been peer reviewed.

Correspondence to: Amit Garg, amit.garg@Ihsc.on.ca

CMAJ Open 2019. DOI:10.9778/cmajo.20190065 
According to eHealth Ontario, as of Dec. 31, 2017, 134 of the 262 hospital sites across the province were using OLIS, and 13 of the 14 Local Health Integration Networks (LHINs) were included. ${ }^{67}$ This variation presents a challenge when conducting retrospective population-based studies to assess laboratory-based outcomes during hospital encounters because, depending on the date of assessment, the patient's laboratory results may or may not be available in OLIS. Thus, if a future study wanted to define a laboratory-based outcome using OLIS and included all hospitals in the province, the study would be biased because only people with laboratory data in OLIS would be captured, and outcomes for those presenting to non-OLIS hospitals would be missed. The aim of the current study was to determine the geographic areas in Ontario from which people would likely have presented to a hospital with laboratory data included in OLIS, and how these areas changed over time. We used the resulting data to construct a date-dependent table of geographic areas likely to have hospital laboratory data available in OLIS for use in future studies in order to accurately assess people presenting to hospitals with laboratory-defined conditions.

\section{Methods}

\section{Study design and research setting}

We conducted this descriptive study using health administrative databases, which are linked through unique encoded identifiers and analyzed at ICES. All Ontario residents receive universal access to physician services. We reported this study according to guidelines for observational studies. ${ }^{8}$

\section{Data sources}

We conducted the study using 6 linked data sets housed at ICES: the Ontario Registered Persons Database, which contains demographic information for all residents of Ontario; the Canadian Institute for Health Information Discharge Abstract Database, which contains hospital admission diagnosis information for all people in Ontario; the National Ambulatory Care Reporting System Database, which contains information about emergency department visits; the OLIS; the Ontario Health Insurance Plan Database, which contains health claims information for both inpatient and outpatient physician services; and the Same Day Surgery Database, which contains information on day-surgery visits in Ontario Additional information about the data sets is provided in Appendix 1 (available at www.cmajopen.ca/content/7/4/E624/ suppl/DC1). The data set from the current study is held securely in coded form at ICES.

\section{Cohort assembly}

We identified all adults (age $\geq 18 \mathrm{yr}$ ) who presented to the emergency department in the National Ambulatory Care Reporting System database for any reason between Apr. 1, 2007, and Dec. 31, 2017. The cohort was classified according to fiscal quarter (Apr. 1-June 30, July 1-Sept. 30, Oct. 1Dec. 31, Jan. 1-Mar. 31). We excluded multiple emergency department visits by an individual patient within each quarter to ensure that only 1 visit per patient per quarter was reported (to avoid correlation by many visits by the same person within a quarter). We excluded emergency department visits if there was missing information on the patient's age or sex, if there was a recorded death date on or before the emergency department visit date, or if the patient was not an Ontario resident. This was a standard data-cleaning exclusion done to ensure we were capturing valid emergency department visits for adults only.

\section{Identifying hospitals linked versus not linked to Ontario Laboratories Information System over time}

Using data from eHealth Ontario, ${ }^{6}$ we compiled a list of Ontario hospitals and the fiscal quarter when they started contributing data to OLIS. To more precisely identify the date contributions began, we searched the OLIS database at ICES for dates of serum creatinine tests from specific hospital laboratories using the unique Canadian Institute for Health Information institution numbers housed at ICES. We chose the serum creatinine test because it is among the top 5 most commonly ordered tests, so seeing when a given hospital began contributing results of this test to OLIS is a good indication of when that hospital joined OLIS. In addition, to date, all the laboratory tests in OLIS have not been linked at ICES, and only certain tests are available, so we chose the most common test that we were familiar with. The actual serum creatinine values were not used in the current study, since we were interested only in measurement dates. We categorized hospitals as OLIS-linked beginning on the date their laboratory data began to populate the OLIS database; they were considered nonlinked before this date. Hospitals that had not contributed data to OLIS before Dec. 31, 2017 were considered nonlinked for the entire study period.

\section{Identifying the Ontario Laboratories Information System catchment area}

In Canada, geographic regions are defined by postal codes, which help postal operators sort and deliver mail. A postal code comprises a combination of 6 characters that identify a delivery unit. The postal code begins with a forward sortation area (FSA; first 3 characters of the postal code). The first character represents the postal district, the second character represents whether the address is urban or rural, and the third character specifies a specific area within a city or town. ${ }^{9}$ The Ontario Registered Persons Database indicated that Ontario had 526 FSAs during the study period.

After determining which hospitals contributed to OLIS and when they started doing so, we sought to determine the geographic areas likely to be served by OLIS-linked hospitals (i.e., the catchment area). To determine a hospital's catchment area, we identified the home location of the patients (based on FSA) presenting to their emergency departments. An FSA was assigned to an OLIS-linked hospital if the hospital received $90 \%$ or more of the emergency department visits arising from that FSA in a given fiscal quarter. We used a 
threshold of $90 \%$ because we wanted to be confident that a majority of emergency department visits that occurred from FSAs within our catchment area would prompt presentation to an OLIS-linked hospital. To apply this threshold, the region must be large enough to calculate stable proportions, but small enough to ensure that misclassification is minimized as much as possible. We considered other geographic units besides FSAs, including dissemination areas, LHINs, subLHINs and census divisions. Dissemination areas are too small, as they consist of 400-700 people, so this group is too sparse to evaluate emergency department visits over a 3-month period. Local Health Integration Networks are too large for defining hospital catchment areas, and even subLHINs have around 150000 people. Also, LHINs are politically created areas that are subject to change over time (and already have been phased out by the provincial government elected in 2018), whereas geographic units based on the census or postal codes are not subject to change based on policy decisions. Although census tracts are a similar boundary, we chose FSAs because they are more commonly used and it is easier to identify people through their actual postal addresses. In addition, FSAs are easily identified and have been used in previous ICES analyses. ${ }^{10}$
Using these criteria, we generated a list of all eligible FSAs and the initial date of joining the catchment area. Next, we produced an interactive Shiny application with a map showing the change in the OLIS catchment area over time using RStudio and the Leaflet package. All other analyses were conducted with SAS version 9.4 (SAS Institute).

\section{Ethics approval}

The use of data in this project was authorized under section 45 of Ontario's Personal Health Information Protection Act, which does not require review by a research ethics board.

\section{Results}

A flow diagram showing catchment area ascertainment between 2007 and 2017 in Ontario is shown in Figure 1. As of Dec. 31, $2017,323(61.4 \%)$ of the 526 FSAs were included in the catchment area (see Appendix 2, available at www.cmajopen.ca/ content/7/4/E624/suppl/DC1, for a list of all eligible FSAs and the date they joined the catchment area). About 8.5 million people resided within the FSAs in the catchment area of OLISlinked hospitals. The baseline characteristics of people residing within the catchment area of OLIS-linked hospitals and those

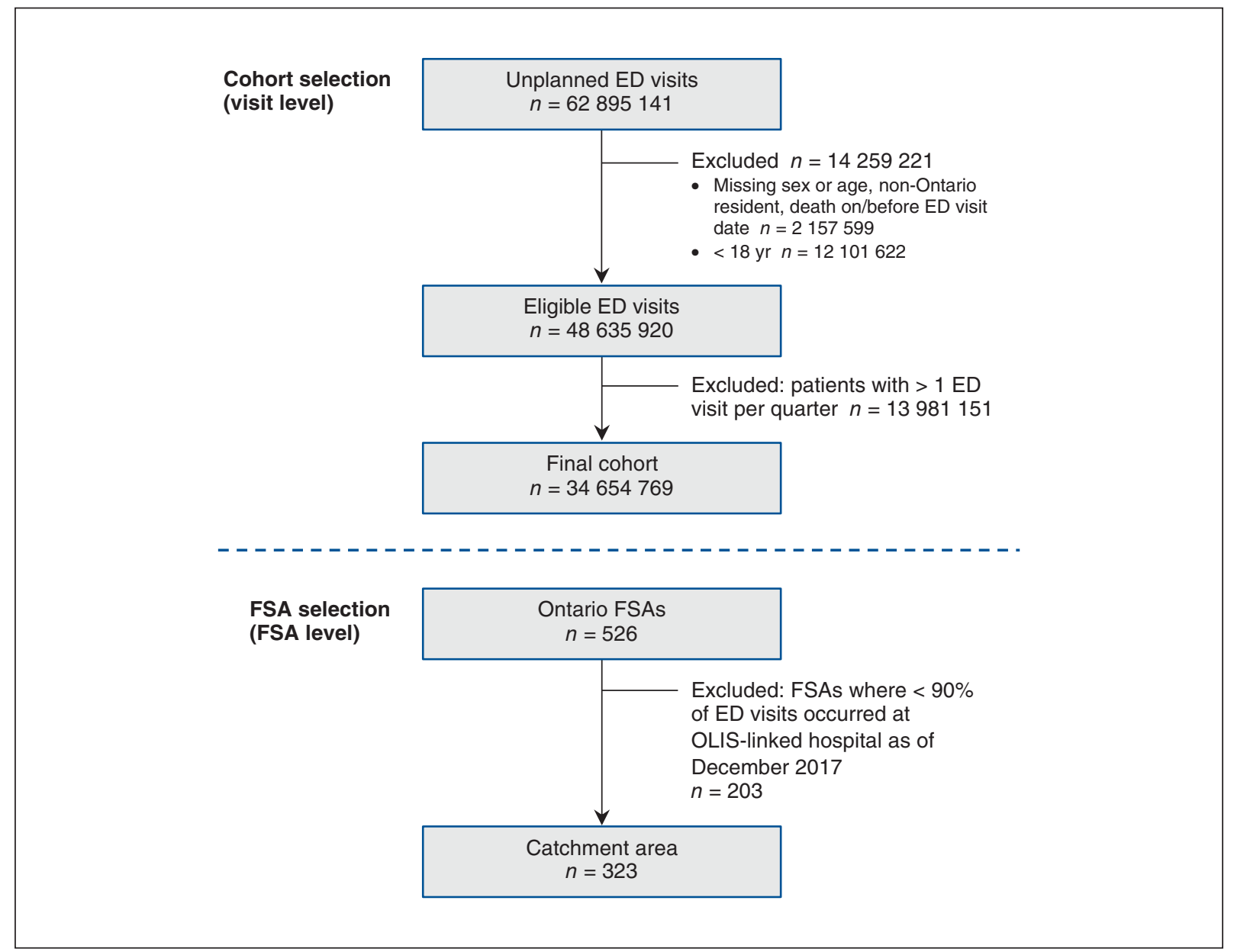

Figure 1: Flow chart showing catchment area ascertainment between Apr. 1, 2007, and Dec. 31, 2017. Note: ED = emergency department, FSA = forward sortation area, OLIS = Ontario Laboratories Information System. 
residing in the catchment area of unlinked hospitals on Dec. 31, 2017 are shown in Table 1 . The 2 groups were similar across selected demographic characteristics and comorbidities.

An interactive map of Ontario is shown at this link: https:// tinyurl.com/OLISmap (see Appendix 3, available at www. cmajopen.ca/content/7/4/E624/suppl/DC1, for explanation of abbreviations). The geographic catchment area continued to grow and expand across the province as more hospitals joined OLIS. A static map of the OLIS catchment area as of Dec. 31, 2017 is depicted in Figure 2.

\section{Interpretation}

In this study, the geographic catchment areas of hospitals with laboratory data available through OLIS were established, along with the temporal changes in these areas. Over time, there was an increase in the number of OLIS-eligible hospitals, with an associated increase in the number of FSAs included in the overall OLIS-linked catchment area. It is reassuring that the characteristics of people within the catchment area are similar to those of people living outside of it, which suggests that results from

\begin{tabular}{|c|c|c|c|}
\hline \multirow[b]{2}{*}{ Characteristic } & \multicolumn{2}{|c|}{ No. $(\%)$ of people* } & \multirow[b]{2}{*}{$\begin{array}{l}\text { Standardized } \\
\text { difference, \%† }\end{array}$} \\
\hline & $\begin{array}{c}\text { Catchment area of } \\
\text { unlinked hospitals } \\
n=4739202\end{array}$ & $\begin{array}{c}\text { Catchment area of } \\
\text { OLIS-linked hospitals } \\
n=8511875\end{array}$ & \\
\hline Female sex & $2380493(50.2)$ & 4318779 (50.7) & 1 \\
\hline Rural residence $\ddagger$ & $514721(10.9)$ & $883021(10.4)$ & 2 \\
\hline \multicolumn{4}{|l|}{ Age, yr } \\
\hline Mean \pm SD & $49.00 \pm 18.52$ & $48.35 \pm 18.24$ & 4 \\
\hline Median (IQR) & 49 (34-62) & $48(33-61)$ & 4 \\
\hline \multicolumn{4}{|l|}{ Age category, yr } \\
\hline $18-34$ & $1260590(26.6)$ & 2309996 (27.1) & 0 \\
\hline $35-44$ & $761956(16.1)$ & $1457822(17.1)$ & 1 \\
\hline $45-54$ & 865243 (18.3) & 1599389 (18.8) & 1 \\
\hline $55-64$ & $825632(17.4)$ & $1434415(16.8)$ & 0 \\
\hline $65-74$ & $567124(12.0)$ & 948119 (11.1) & 1 \\
\hline $75-84$ & $303503(6.4)$ & 500634 (5.9) & 1 \\
\hline $85-94$ & $132614(2.8)$ & $219658(2.6)$ & 1 \\
\hline$\geq 95$ & $22540(0.5)$ & $41842(0.5)$ & 0 \\
\hline \multicolumn{4}{|l|}{ Income quintile§ } \\
\hline 1 (lowest) & $998131(21.1)$ & 1539627 (18.1) & 7 \\
\hline 2 & 944928 (19.9) & $1629030(19.1)$ & 2 \\
\hline 3 & 920995 (19.4) & $1741443(20.4)$ & 3 \\
\hline 4 & $929142(19.6)$ & $1851508(21.8)$ & 5 \\
\hline 5 (highest) & $946006(20.0)$ & 1750267 (20.6) & 1 \\
\hline \multicolumn{4}{|c|}{ Comorbidities in previous $5 \mathrm{yr}$} \\
\hline Hypertension & $1084337(22.9)$ & $1863194(21.9)$ & 2 \\
\hline Diabetes & $541778(11.4)$ & $1011328(11.9)$ & 1 \\
\hline Chronic kidney disease & $144924(3.1)$ & $243818(2.9)$ & 1 \\
\hline Congestive heart failure & $112954(2.4)$ & $164019(1.9)$ & 3 \\
\hline Major cancerף & $201358(4.2)$ & $357584(4.2)$ & 0 \\
\hline \multicolumn{4}{|c|}{$\begin{array}{l}\text { Note: IQR = interquartile range, OLIS = Ontario Laboratories Information System, SD = standard deviation. } \\
\text { *Except where noted otherwise. } \\
\text { †Standardized differences are less sensitive to sample size than traditional hypothesis tests. They provide a measure of the } \\
\text { difference between the groups divided by the pooled standard deviation; a value greater than } 10 \% \text { is interpreted as a } \\
\text { meaningful difference between the groups. }{ }^{11} \\
\text { †Missing rural status was categorized as not rural. } \\
\text { \$Missing income quintile was imputed into the third quintile. } \\
\text { qLung/bronchi, colon/rectum, breast, pancreas, prostate, leukemia, non-Hodgkin lymphoma, liver, ovarian or esophageal. }\end{array}$} \\
\hline
\end{tabular}




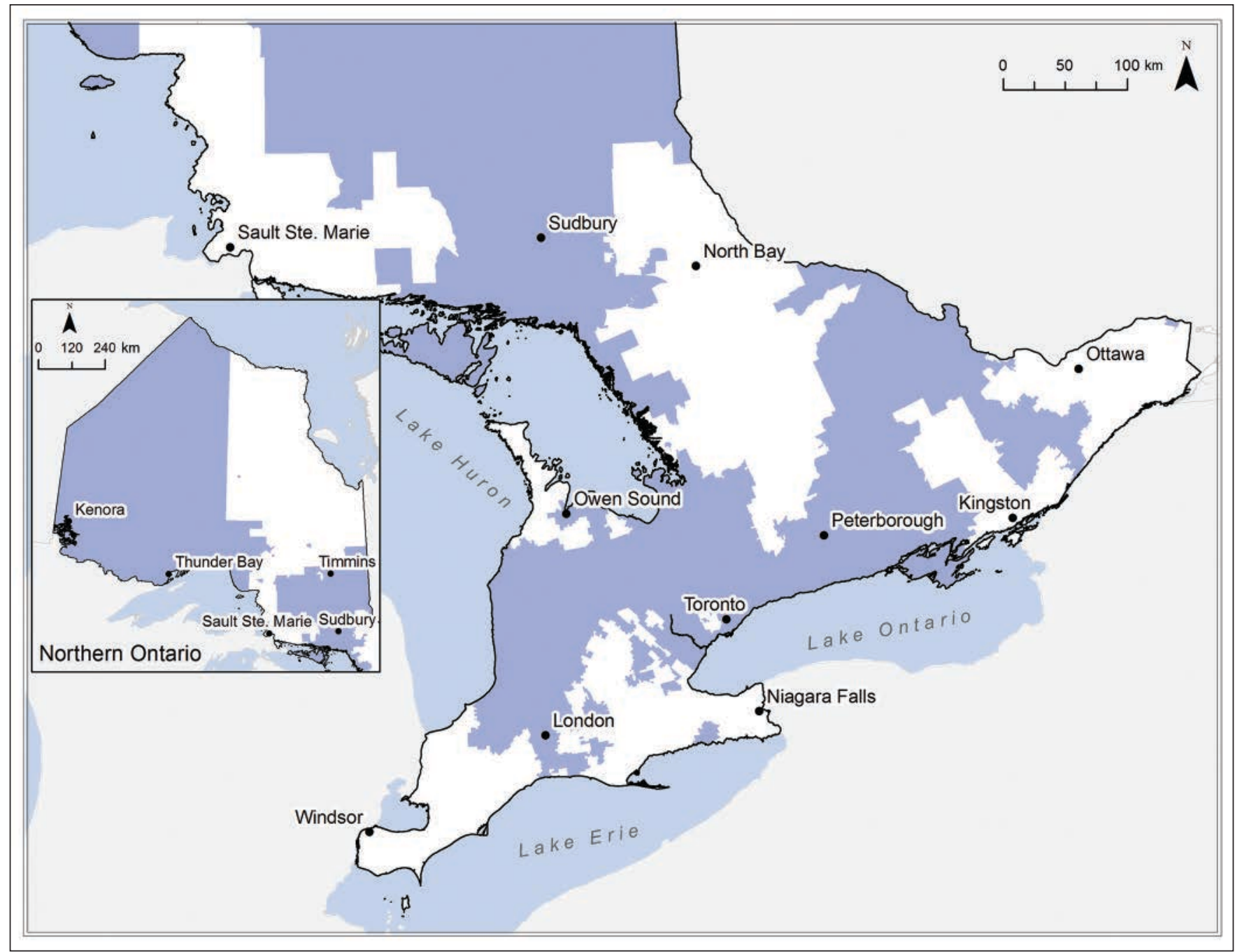

Figure 2: Representation of all catchment regions (blue shading) eligible for the Ontario Laboratories Information System as of Dec. 31, 2017.

future studies restricted to the OLIS catchment area should be generalizable to the entire Ontario population. People will be included in these future cohorts examining laboratory-based outcomes in the hospital setting only if their cohort entry dates were on or before the OLIS eligibility dates in the table of all eligible FSAs and the date they joined the catchment area.

This study builds on previous work done in Ontario that used common electronic medical laboratory data from 12 hospitals to define geographic catchment regions within southwestern Ontario (Cerner system). ${ }^{10}$ Since that paper was published, there have been a number of population-based cohort studies using the defined Cerner catchment area that assessed the risk for hospital admission for rhabdomyolysis after statin use, the risk of acute kidney injury after coprescription of clarithromycin compared to azithromycin in patients taking a calcium-channel blocker, and hyponatremia after the use of antidepressant and antiepileptic drugs. ${ }^{12-15}$

\section{Strengths and limitations}

A strength of the current study is our use of emergency department visits to establish hospital catchment areas rather than inpatient hospital admission, which may not represent the local hospital closest to the patient, as people may travel farther to receive specialized services. This method reduced the risk of outcome misclassification bias, as we likely captured a set of eligible FSAs for a particular hospital that a patient would present to. The reliability of our findings is supported by the large number of emergency department visits across the entire province to form the basis of catchment area ascertainment. In addition, there were few eligibility restrictions, as all adults permanently residing in Ontario were considered for study inclusion. A limitation to the approach of using emergency department visits to establish hospital catchment areas is that some people who are transferred and admitted to a hospital outside their local catchment area will not be captured. In addition, there were discrepancies between the fiscal quarter dates provided by the eHealth Ontario website and the dates that laboratory tests first appeared in OLIS data. Another limitation was that we determined catchment areas using a threshold of at least $90 \%$ of people from a given FSA with an emergency department visit presenting at an OLISlinked hospital, so not all people residing in a catchment area 
visited an OLIS-linked hospital. This introduces the small risk of nondifferential outcome misclassification bias for future studies that use this catchment area to define their study populations, which may underestimate the true effect. We also recognize that certain readers may prefer a lower threshold to include more people, but, for our purposes at ICES and in our future studies, we wanted to be confident we were assessing outcomes correctly, rather than have larger samples with a higher risk of outcome misclassification bias. In addition, there may have been some loss of information from dichotomizing at $90 \%$ rather than using the actual percentages for all FSAs. However, we chose to dichotomize the data because, to create the catchment area, we needed a specified threshold to use. The threshold of $90 \%$ maximizes specificity (i.e., minimizes false-positive FSAs) while potentially hindering sensitivity. It is worth noting that the catchment area would potentially change if we had included all emergency department records and not just the first 1 per person per quarter, but we decided to use an approach whereby there would be no within-individual correlation within the quarters. We treated each quarter as a different "cohort" and then linked the FSAs from each of these cohorts to OLIS or nonOLIS hospitals; thus, no direct comparison or testing occurred between quarters, so we felt no need to account for this correlation. Another consideration is that hospitals within Ontario are continually joining OLIS; thus, updates to this catchment area will be needed in the future.

\section{Conclusion}

Researchers who wish to use OLIS in future studies may use the date-dependent table for ascertaining laboratorydiagnosed outcomes, such as acute kidney injury related to drug exposure, among patients admitted to hospital. The results of the current study can be used in future research to assess OLIS-linked laboratory outcomes by ensuring that people included in these future cohorts are accurately captured in OLIS.

\section{References}

1. Goel V, Williams J, Anderson G, et al. Patterns of health care in Ontario: the ICES practice atlas. 2nd ed. Ottawa: Canadian Medical Association; 1996.

2. Hwang YJ, Shariff SZ, Gandhi S, et al. Validity of the International Classification of Diseases, Tenth Revision code for acute kidney injury in elderly patients at presentation to the emergency department and at hospital admission. BMF Open 2012;2.pii:e001821.

3. Fleet JL, Shariff SZ, Gandhi S, et al. Validity of the International Classification of Diseases 10th revision code for hyperkalaemia in elderly patients at presentation to an emergency department and at hospital admission. BMF Open 2012;2.pii: e002011.

4. Gandhi S, Shariff SZ, Fleet JL, et al. Validity of the International Classification of Diseases 10th revision code for hospitalisation with hyponatraemia in elderly patients. BM7 Open 2012;2.pii:e001727.

5. eHealth Ontario. Toronto: Ontario Laboratories Information System. Available: https://www.ehealthontario.on.ca/en/for-healthcare-professionals/ ontario-laboratories-information-system-olis (accessed 2019 Oct. 8).

6. OLIS: data contributors. Toronto: Ontario Laboratories Information System; 2018:9-10.

7. Your hospitals. Toronto: Ontario Hospital Association. Available: https://www. oha.com/your-hospitals (accessed 2019 Feb. 11).
8. Benchimol EI, Smeeth L, Guttmann A, et al. The REporting of studies Conducted using Observational Routinely-collected health Data (RECORD) statement. PLOS Med 2015;12:e1001885.

9. Forward sortation areas - the first segment of the postal code. In: Addressing guidelines: Canada postal guide. Ottawa: Canada Post; 2019. Available: https:// www.canadapost.ca/tools/pg/manual/PGaddress-e.asp\#1449304 (accessed 2018 May 15).

10. Gandhi S, Shariff SZ, Beyea MM, et al. Identifying geographical regions serviced by hospitals to assess laboratory-based outcomes. BM7 Open 2013;3.pii: e001921.

11. Austin PC. Using the standardized difference to compare the prevalence of a binary variable between two groups in observational research. Commun Stat Comput Simulation Comput 2009;38:1228-34.

12. Patel AM, Shariff S, Bailey DG, et al. Statin toxicity from macrolide antibiotic coprescription: a population-based cohort study. Ann Intern Med 2013;158: 869-76.

13. Gandhi S, Fleet JL, Bailey DG, et al. Calcium-channel blocker-clarithromycin drug interactions and acute kidney injury. FAMA 2013;310:2544-53.

14. Gandhi S, Shariff SZ, Al-Jaishi A, et al. Second-generation antidepressants and hyponatremia risk: a population-based cohort study of older adults. Am 7 Kidney Dis 2017;69:87-96.

15. Gandhi S, McArthur E, Mamdani MM, et al. Antiepileptic drugs and hyponatremia in older adults: two population-based cohort studies. Epilepsia 2016;57: 2067-79.

Affiliations: Departments of Epidemiology and Biostatistics (Iskander, Nash, Muanda, Garg) and Medicine (Weir), Western University, London, Ont.; ICES (McArthur, Nash, Gandhi-Banga, Weir, Muanda, Garg); Epidemiology and Benefit-Risk Evaluation (Gandhi-Banga), Sanofi Pasteur, Toronto, Ont.

Contributors: Amit Garg conceived of the study. Carina Iskander, Eric McArthur, Danielle Nash, Sonja Gandhi-Banga and Amit Garg designed the study. Eric McArthur analyzed the data. Carina Iskander drafted the manuscript, and Eric McArthur, Danielle Nash, Sonja Gandhi-Banga, Matthew Weir, Flory Tsobo Muanda and Amit Garg critically revised it for important intellectual content. All of the authors interpreted the data, approved the final version to be published and agreed to be accountable for all aspects of the work.

Funding: This study was supported by the ICES Western site. ICES is funded by an annual grant from the Ontario Ministry of Health and Long-Term Care (MOHLTC). Core funding for ICES Western is provided by the Academic Medical Organization of Southwestern Ontario, the Schulich School of Medicine and Dentistry, Western University and the Lawson Health Research Institute. The research was conducted by members of the ICES Kidney, Dialysis and Transplantation team, at the ICES Western facility, who are supported by a grant from the Canadian Institutes of Health Research (CIHR). Amit Garg was supported by the Dr. Adam Linton Chair in Kidney Health Analytics and a CIHR Clinician Investigator Award.

Acknowledgement: The authors thank Alexandra Ouédraogo, at ICES Western, London, Ontario, for her graphic work.

Disclaimer: This study was supported by ICES, which is funded by an annual grant from the Ontario Ministry of Health and Long-Term Care (MOHLTC). The opinions, results and conclusions reported in this article are those of the authors and are independent from the funding sources. No endorsement by ICES, the Academic Medical Organization of Southwestern Ontario, the Schulich School of Medicine and Dentistry, the Lawson Health Research Institute, the Canadian Institutes of Health Research or the MOHLTC is intended or should be inferred.

Supplemental information: For reviewer comments and the original submission of this manuscript, please see www.cmajopen.ca/content/7/4/ E624/suppl/DC1. Although data-sharing agreements prohibit ICES from making the data set for the current study publicly available, access may be granted to those who meet prespecified criteria for confidential access, available at www.ices.on.ca/DAS. The full data set creation plan and underlying analytic code are available from the authors on request, with the understanding that the programs may rely on coding templates or macros that are unique to ICES. 